\title{
Design of the New Experimental Storage Ring for operation with ions and antiprotons
}

\author{
C. Dimopoulou, K. Beckert, P. Beller,* A. Dolinskii, U. Laier, F. Nolden, G. Schreiber, M. Steck, and J. Yang \\ Gesellschaft für Schwerionenforschung, Planckstrasse 1, 64291, Darmstadt, Germany
}

(Received 28 September 2006; published 12 February 2007)

\begin{abstract}
The New Experimental Storage Ring (NESR) of the Facility for Antiproton and Ion Research is designed for versatile operation with ions and antiprotons. Both stable ions and rare isotope beams will be used for internal experiments. The ion beams can be decelerated to a minimum energy of $4 \mathrm{MeV} / u$ and extracted to a low energy experimental area. Antiprotons will be decelerated from $3 \mathrm{GeV}$ to a minimum energy of $30 \mathrm{MeV}$ and transferred to the Facility for Low-energy Antiproton and Ion Research. Electron cooling will provide high quality beams for experiments, assist the deceleration process, and allow the accumulation of rare isotope beams by stacking in longitudinal phase space. Specific rf systems are foreseen for the various operation modes.
\end{abstract}

DOI: 10.1103/PhysRevSTAB.10.020101

PACS numbers: 29.20.Dh, 29.27.Bd, 41.75.-i

\section{INTRODUCTION}

The major modes of operation of the New Experimental Storage Ring (NESR) of the Facility for Antiproton and Ion Research (FAIR) [1] will be (i) storage of highly charged radioactive and stable ion beams in the energy range 740 to $4 \mathrm{MeV} / u$ for a variety of internal atomic and nuclear physics experiments and (ii) efficient deceleration of ions and antiprotons before transfer to a low energy experimental area. Originally, the NESR was proposed also for accumulation of antiprotons [1,2]. Because of severe constraints on the ion optical lattice and lack of space for stochastic cooling components, this idea was abandoned and the Recycled Experimental Storage Ring (RESR) was added to the FAIR concept as a dedicated accumulator ring for antiprotons [3]. Operation in both magnet polarities, with ions and antiprotons, is required.

Ion beams can be injected either directly from the synchrotrons SIS18 or SIS100 [4], where they are accelerated to the required energy, or as secondary rare isotope beams (RIBs) emerging from a production target and selected in the magnetic separator SuperFRS [5]. There will be two ways of injecting the RIBs into the NESR. One is to inject directly from the SuperFRS. A better injection beam quality is reached after precooling in the Collector Ring (CR) [6]. Then, in the RESR the beam can be optionally decelerated and transferred to the NESR. The general layout of the storage rings of FAIR is shown in Fig. 1. At the maximum injection energy of $740 \mathrm{MeV} / u$, the ions will be completely stripped. Injection at lower energy is also foreseen, if lower energy or lower charge states are required by experiments. For the planned collider mode, fast deceleration of the ions from $740 \mathrm{MeV} / u$ to the required energy in the range 740 to $100 \mathrm{MeV} / u$ can take place in the RESR [3].

Deceleration of ions to a minimum energy of $4 \mathrm{MeV} / u$ in the NESR requires a reduction of the magnetic field by a

\footnotetext{
*Deceased.
}

factor of 25. The maximum ramp rate of $1 \mathrm{~T} / \mathrm{s}$ will allow a change of the bending field between 1.6 and $0.06 \mathrm{~T}$ within $1.5 \mathrm{~s}$. This is particularly important for experiments with short-lived RIBs.

After stochastic precooling in the $\mathrm{CR}$ and accumulation in the RESR, up to $1 \times 10^{9}$ antiprotons will be injected into the NESR at an energy of $3 \mathrm{GeV}$ and decelerated to a minimum energy of $30 \mathrm{MeV}$. The main user of decelerated ions and antiprotons will be the Facility for Low-energy Antiproton and Ion Research (FLAIR) [7] of the FAIR complex. FLAIR requires a short bunch of approximately $30 \mathrm{~m}$ length (i.e. $15 \%$ of the ring circumference) of ions at $4 \mathrm{MeV} / u$ and antiprotons at $30 \mathrm{MeV}$.

A powerful electron cooling system will provide highest phase space density of the stored beams and, consequently, optimum beam quality for the experiments. It will cover the full energy range ( 740 to $4 \mathrm{MeV} / u$ ) for ions and allows intermediate cooling in the range 800 to $30 \mathrm{MeV}$ during the deceleration of antiprotons. Electron cooling will also

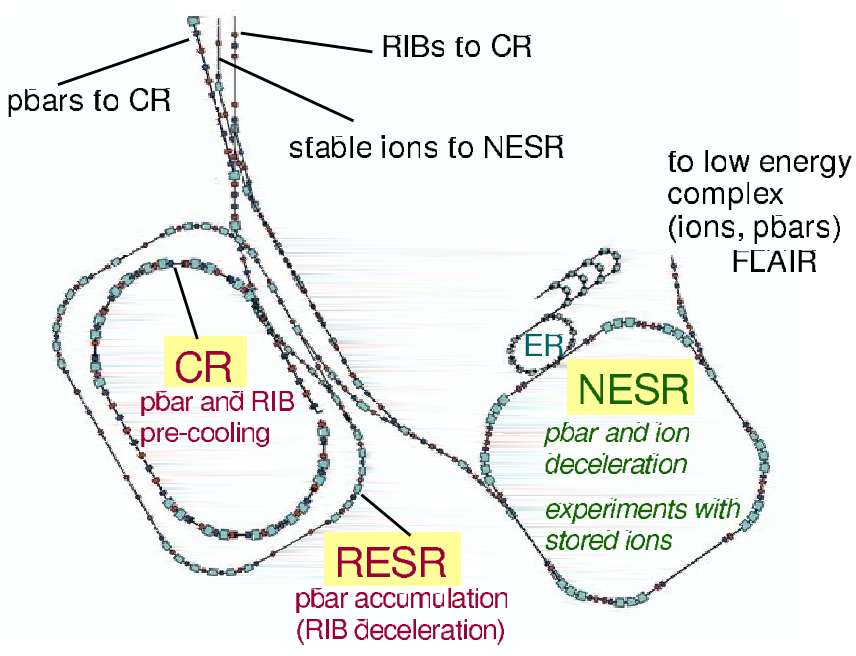

FIG. 1. (Color) The $13 \mathrm{Tm}$ storage rings of the FAIR facility for ions and antiprotons. 


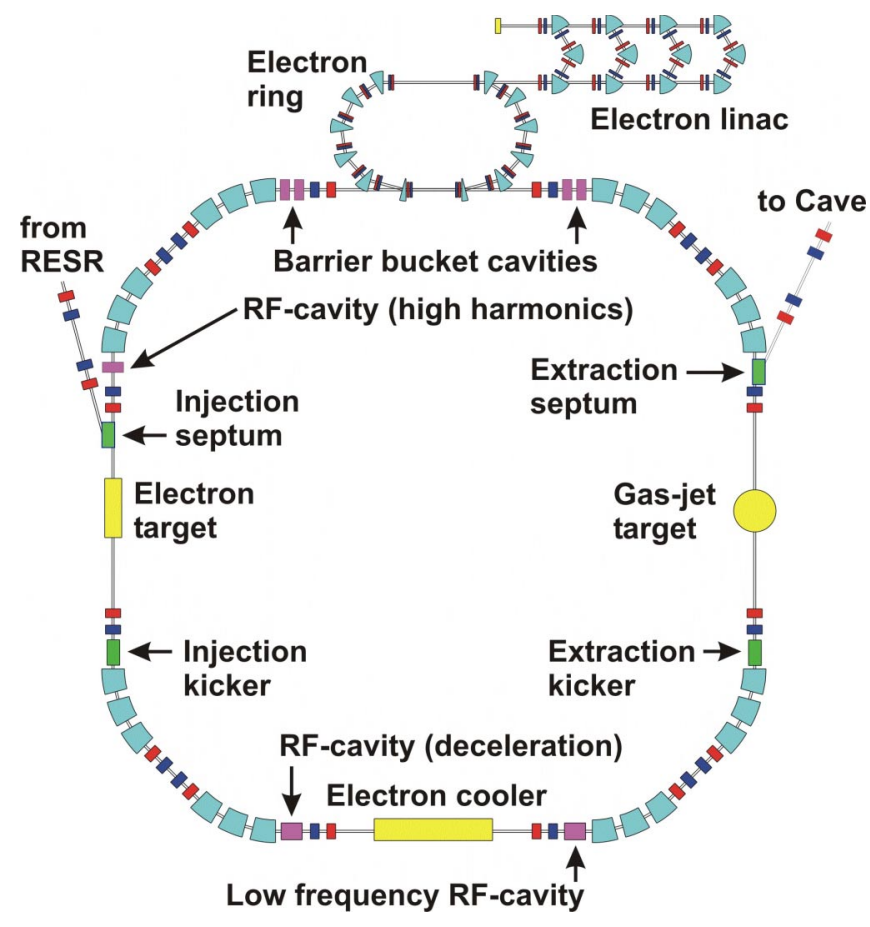

FIG. 2. (Color) General layout of the NESR storage ring.

compensate the diffusion (blowup) of the beams during deceleration and thus high efficiency and low losses can be achieved. In addition, electron cooling will support the accumulation (longitudinal compression) of RIBs which can be performed at injection energy, in the range $100-740 \mathrm{MeV} / u$. For experiments with short-lived isotopes, the cooling time and the time of deceleration will be optimized to a few seconds. The electron cooler will be installed in one of the 4 straight sections of the NESR, as shown in Fig. 2. The central parts of the remaining three straight sections will be mainly used for experimental installations.

An internal target, either a gas jet, cluster or a pellet target, will allow a large variety of atomic and nuclear physics experiments. The pellet target provides higher density and is particularly suited for nuclear physics stud- ies with radioactive ions, where high luminosity is required. Electron cooling will compensate the heating of the beam in the internal target. Detector setups will surround the interaction volume. Projectilelike particles scattered within the acceptance will be detected downstream from the interaction point.

An electron target, which is largely a second electron cooler operating at lower electron energies up to $40 \mathrm{keV}$, will provide cold electrons at variable relative velocity for atomic physics recombination experiments $[8,9]$. It can also be used as an electron cooler for decelerated ion beams up to an energy of $73 \mathrm{MeV} / u$, in a regime where it will be superior to the standard electron cooler. In turn, the electron cooler can serve as an experimental setup for recombination experiments at higher electron beam energies than those accessible by the electron target.

After a first preliminary design [10], one straight section is still being optimized as a collision section between electrons of up to $500 \mathrm{MeV}$ and cooled RIBs. The electrons will be provided by a complex consisting of an electron linac followed by an electron ring (ER).

As a future option, it has also been proposed to decelerate antiprotons in the RESR to $125 \mathrm{MeV}$ and then store them in the electron ring for collision experiments. The final layout of the electron ring and of the interaction region are still under study.

For precision experiments with stored ion beams, an excellent stability of all components is required. For instance, mass measurements of rare isotopes require a stability of the revolution frequency of $10^{-7}$ or better.

The operation with low energy or partially stripped ions as well as the deceleration of antiprotons impose stringent requirements on the vacuum system. In order to reach a basic pressure below $10^{-11}$ mbar, the whole vacuum system has to be bakable at a maximum temperature of $300^{\circ} \mathrm{C}$ and all vacuum components have to be compatible with ultrahigh vacuum standards.

\section{ION OPTICAL LATTICE}

The ion optical parameters of the storage ring, described in [11], have been refined. They are summarized in Table I.

TABLE I. NESR ring parameters.

\begin{tabular}{lc}
\hline \hline Circumference & $222.11 \mathrm{~m}$ \\
Maximum magnetic rigidity $B \rho$ & $13 \mathrm{Tm}$ \\
Minimum bending power after deceleration & $0.55 \mathrm{Tm}$ \\
Maximum $A / Z$ & 2.7 \\
Horizontal/vertical acceptance $(\Delta p / p=0)$ & $460 / 55 \mathrm{~mm} \mathrm{mrad}$ \\
Horizontal/vertical acceptance $(\Delta p / p= \pm 1.5 \%)$ & $160 / 50 \mathrm{~mm} \mathrm{mrad}$ \\
Momentum acceptance $\left(\epsilon_{H}=0\right)$ & $\pm 2.1 \%$ \\
Horizontal/vertical tune & $3.4 / 3.2$ \\
Transition energy $\gamma_{t}$ & 5.74 \\
Maximum dispersion & $7.24 \mathrm{~m}$ \\
Vacuum pressure & $\leq 10^{-11} \mathrm{mbar}$ \\
\hline \hline
\end{tabular}




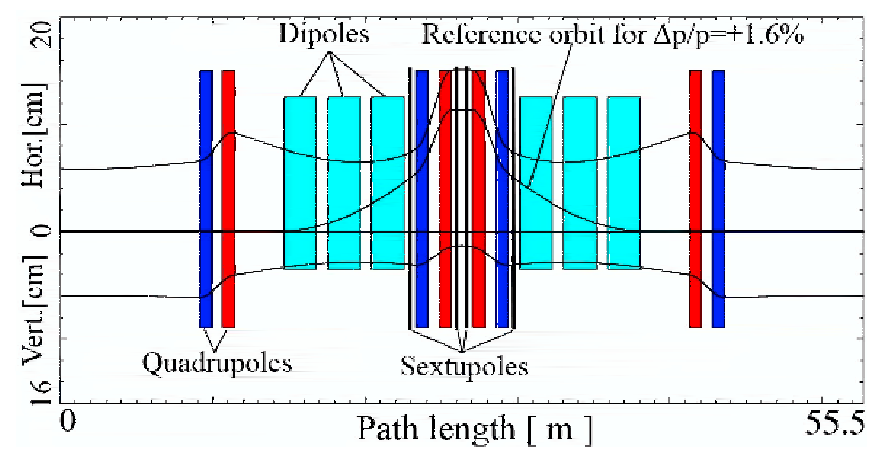

FIG. 3. (Color) Beam envelopes and reference orbit for $\Delta p / p=$ $+1.6 \%$ in one quarter of the ring.

The ring circumference will be $222.11 \mathrm{~m}$ with four $18 \mathrm{~m}$ long straight sections in a basic lattice with fourfold symmetry based on doublet focusing. Figure 3 shows one quarter of the lattice structure of the NESR, the corresponding beam envelopes as well as the reference orbit for particles with a momentum deviation $\Delta p / p=+1.6 \%$ (which is proportional to the dispersion function). The ion optics calculations have been made with the MIRKO code [12].

The maximum strength of the main magnetic ring elements up to a bending power of $13 \mathrm{Tm}$ can be realized using normal conducting magnet technology. The option of superferric dipole magnets, which is expected to reduce the operation costs of these large aperture magnets, is under investigation. For a maximum momentum deviation of $\pm 1.5 \%$, the ring lattice offers a transverse acceptance of

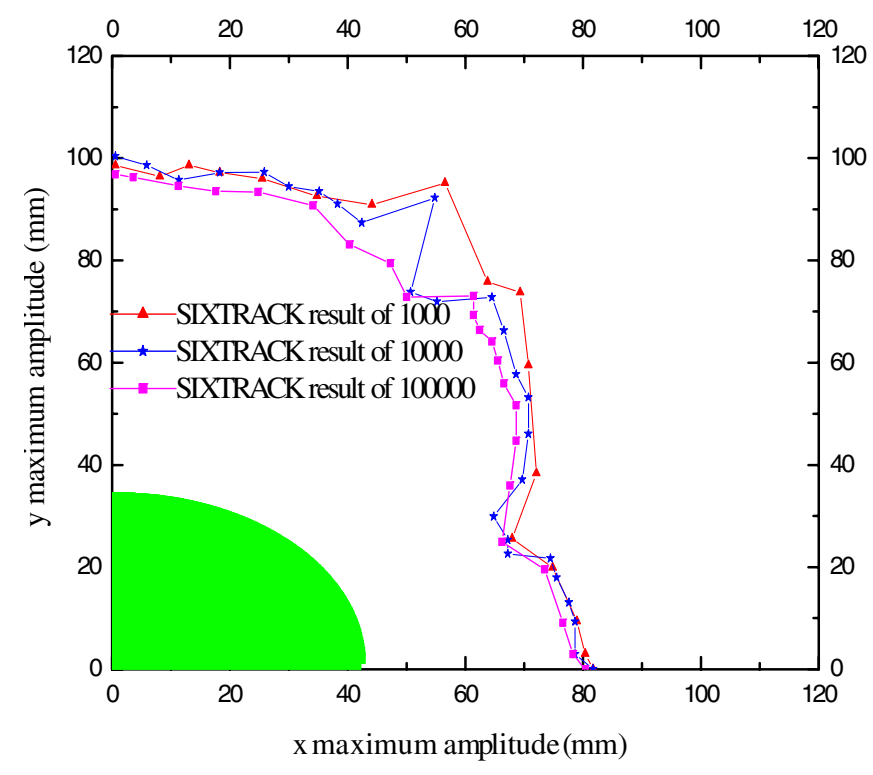

FIG. 4. (Color) Dynamic aperture calculation with SIXTRACK for particles with a momentum deviation of $1.5 \%$. The dynamic aperture, which was tracked over different numbers of turns $(1000,10000,100000)$, is much larger than the acceptance of the ring (shaded green area).
$160 \mathrm{~mm}$ mrad horizontally and $50 \mathrm{~mm}$ mrad vertically. The dynamic aperture has been calculated (i) including the sextupole magnets for the chromaticity correction and (ii) assuming the maximum higher order components of dipole and quadrupole magnets, according to previous experience with large aperture magnets at GSI. The results of such calculations with the SIXTRACK [13] and the PTC [14] codes confirmed that the dynamic aperture significantly exceeds the size of a beam filling the full acceptance. The result of a SIXTRACK calculation is shown in Fig. 4 for optimized tunes of $Q_{H}=3.37$ and $Q_{V}=3.18$ and for various numbers of turns $(1000,10000$, and 100000$)$.

The large momentum acceptance allows simultaneous storage of beam components with a large momentum deviation for experiments, e.g., different charge states of highly charged ions, rare isotopes with different charge to mass ratios, or products of internal reactions with large momentum change. Intercepting particle detectors installed in the bending sections will allow detection of particles produced in the straight sections. Thus, the bending sections can be used as spectrometers for reaction products.

\section{INJECTION AND EXTRACTION}

The beams to be stored in the NESR will be injected as a single bunch with a length between $20 \%$ and $80 \%$ of the ring circumference. The short bunch length of the injected beam allows adiabatic debunching in the ring in order to reduce the momentum spread for fast cooling.

Three full aperture kicker magnets with a rise and fall time below $100 \mathrm{~ns}$ are foreseen [1]. Their polarity can be changed. They will allow injection of hot RIBs directly from the fragment separator SuperFRS. The fast rise and fall time is advantageous because, depending on the beam velocity, up to $80 \%$ of the ring circumference is available for injection or a longitudinal accumulation method (see Sec. VI). The latter is particularly important in order to increase the intensity of RIBs.

Since only decelerated beams will be extracted, the magnetic rigidity of the extraction components can be limited to $4 \mathrm{Tm}$ and the required field strengths are moderate. Since these beams are cooled, a small aperture magnetic or an electrostatic septum will be used. Slow resonant extraction by changing the tune or by rf noise will be available.

\section{ELECTRON COOLING}

The required beam quality for precision experiments with stored ion beams will be achieved by electron cooling. Electron cooling will also support all beam manipulations involving rf systems, like beam accumulation and deceleration. The small momentum spread of the cooled beam will relax the requirements on the rf systems. For the antiprotons an intermediate cooling is foreseen after de- 
TABLE II. NESR electron cooler parameters.

\begin{tabular}{lc}
\hline \hline Energy range & $2-450 \mathrm{keV}$ \\
Maximum voltage & $500 \mathrm{kV}$ \\
Voltage ramping rate & $250 \mathrm{kV} / \mathrm{s}$ \\
Maximum current & $2 \mathrm{~A}$ \\
Length of cooling section & $5 \mathrm{~m}$ \\
Cathode radius & $1 \mathrm{~cm}$ \\
Beam radius in cooling section & $0.5-1.4 \mathrm{~cm}$ \\
Magnetic field: & \\
Gun & up to $0.4 \mathrm{~T}$ \\
Cooling section & up to $0.2 \mathrm{~T}$ \\
Collector & up to $0.23 \mathrm{~T}$ \\
Straightness $B_{\perp} / B_{\|}$ & $\leq 5 \times 10^{-5}$ \\
Maximum power in the collector & $15 \mathrm{~kW}$ \\
\hline \hline
\end{tabular}

celeration from $3 \mathrm{GeV}$ to $800 \mathrm{MeV}$. Therefore, a cooling system for a range of electron energies from 2 to $450 \mathrm{keV}$ has been designed [15]. Its parameters are given in Table II. A homogeneous electron beam with variable diameter for an optimal overlap with the different ion beam sizes can be produced by adiabatic expansion or compression of the guiding longitudinal magnetic field. The option of a hollow electron beam, which seems to be advantageous for reducing ion-electron recombination losses, is also included. A straightness of the magnetic field lines better than $B_{\perp} / B_{\|}=5 \times 10^{-5}$ at a maximum strength of $0.2 \mathrm{~T}$ and a fast ramping within $1.5 \mathrm{~s}$ during beam deceleration are stringent requirements on the magnet system. The magnetic field of the electron cooler needs to be ramped because it creates a local orbit distortion inversely proportional to the ion beam rigidity $B \rho$, which has to be compensated. Electron currents of up to 2 A will allow fast cooling of short-lived isotopes. Short cooling times will crucially depend on the beam parameters after precooling in the CR and RESR storage rings. This is particularly important because of the high beam velocity.

A special requirement for the electron cooling system is fast ramping of the high voltage during deceleration. Whereas cooling after injection for ions and at the intermediate energy for antiprotons requires electron energies around $400 \mathrm{keV}$, after deceleration the electron energy must be lowered to a few $\mathrm{keV}$. As a consequence, an active method of discharging and charging the high voltage section is required to be able to ramp the accelerating voltage in $1.5 \mathrm{~s}$ between upper and lower values.

The dependence of the cooling time on the initial beam parameters has been demonstrated by simulations with the BETACOOL code [16]. The simulations confirmed the necessity of a magnetic field strength of $0.2 \mathrm{~T}$ and a field straightness in the cooling section of $B_{\perp} / B_{\|}=5 \times 10^{-5}$ for fast cooling. For ion beams at the maximum injection energy of $740 \mathrm{MeV} / u$, cooling times of less than $0.5 \mathrm{~s}$ were calculated, if the precooled beam from the $\mathrm{CR}$ storage ring is cooled with an electron beam of $1 \mathrm{~A}$. An example of

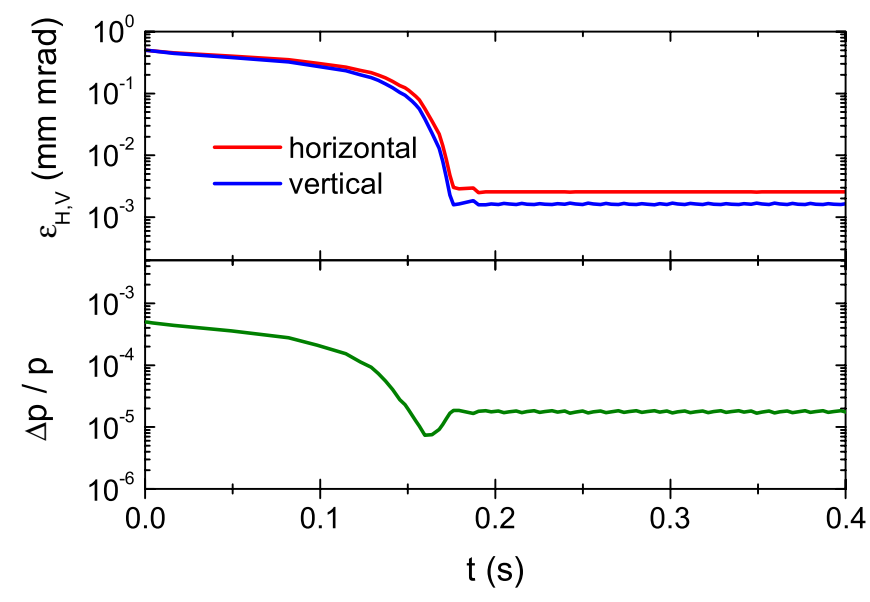

FIG. 5. (Color) Simulations of electron cooling of a coasting beam of $10^{8}{ }^{132} \mathrm{Sn}^{50+}$ ions at $740 \mathrm{MeV} / u$. The initial beam parameters correspond to the precooled beam from $\mathrm{CR}$. Electron beam density: $3.2 \times 10^{8} \mathrm{~cm}^{-3}$ (1 A, $5 \mathrm{~mm}$ beam radius). Magnetic field in the cooling section: $B=0.2 \mathrm{~T}$.

such simulations is shown in Fig. 5 for ${ }^{132} \mathrm{Sn}^{50+}$ ions at $740 \mathrm{MeV} / u .{ }^{132} \mathrm{Sn}^{50+}$ are chosen as reference ions because they (i) are neutron-rich nuclei interesting for experiments and (ii) have the maximum design ratio $A / Z=2.7$ of NESR at an intermediate charge state. In all simulations, Parkhomchuk's empirical formula [17] with an effective electron velocity corresponding to magnetic field misalignments of $5 \times 10^{-5}$ is used for the cooling force and the Martini model [18] for intrabeam scattering (IBS). As the phase space occupied by the beam shrinks due to cooling, the IBS heating rate increases, and finally equilibrium values of emittance and momentum spread are reached. This can be clearly seen in Fig. 5, where cooling in the longitudinal direction proceeds faster than in the transverse and, after low momentum spreads are reached, IBS heats the beam up towards equilibrium. Even if the momentum spread and the emittance are larger by a factor of 2 than the design value of the $\mathrm{CR}$, the cooling time is not expected to exceed $1.5 \mathrm{~s}$. Thus, storage ring experiments with RIBs can benefit without restrictions from the planned cycle time of $1.5 \mathrm{~s}$ of the synchrotron SIS100.

The conditions for electron cooling are less favorable for antiprotons which are injected at $3 \mathrm{GeV}$ and have larger momentum spread and emittance [6]. Because of their low charge, the cooling time easily approaches the order of minutes. The dependence of the cooling time on momentum spread and emittance for antiprotons injected at $3 \mathrm{GeV}$ and decelerated in the NESR to $800 \mathrm{MeV}$ is shown in Fig. 6. An adiabatic growth of the beam parameters is assumed during the deceleration in the NESR. The cooling time for antiprotons with a deviation corresponding to the $1 \sigma$-value of the distribution after stochastic precooling in the CR is $150 \mathrm{~s}$, even assuming an electron beam current of 2 A. Clearly, if a deceleration cycle time below $1 \mathrm{~min}$ is requested by experiments with low energy antiprotons, 


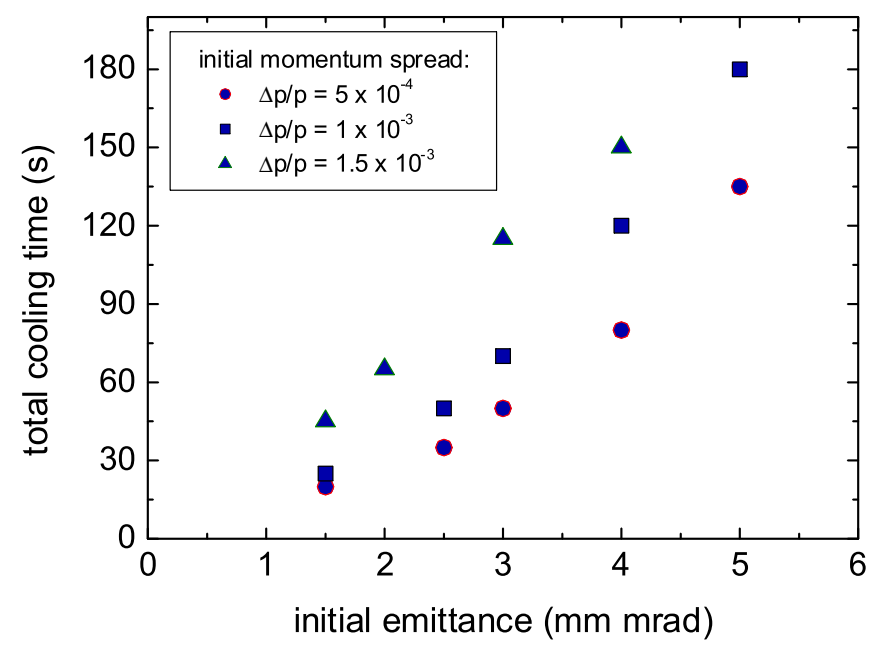

FIG. 6. (Color) Total cooling time for antiprotons at $800 \mathrm{MeV}$ as a function of transverse emittance and for different initial momentum spreads.

better precooling must be achieved. This should be possible by additional stochastic cooling of the antiprotons in the accumulator ring RESR. Beam dynamics studies are underway in order to optimize the beam quality in the RESR.

During the deceleration process, electron cooling at intermediate energies will be applied. The equilibrium parameters of the ion and antiproton beams have been simulated with BETACOOL, for realistic operation parameters of the electron cooler. They can be taken into account in considerations on the rf systems (see also Sec. V) and the experiments. The results for the injection and extraction energies are given in Table III.

Taking into account the space charge limitations for highly charged ions at the lowest energy of $4 \mathrm{MeV} / u$, a maximum intensity of $5 \times 10^{7}$ cooled ions is estimated, if the equilibrium emittance does not exceed $1 \mathrm{~mm}$ mrad. The

TABLE III. Equilibrium parameters ( $2 \sigma$ values) for electroncooled ion and antiproton beams in the NESR.

\begin{tabular}{cccc}
\hline \hline \multicolumn{4}{c}{ Coasting beam of $10^{8}{ }^{238} \mathrm{U}^{92+}$} \\
\hline $\begin{array}{c}\text { Kinetic energy } \\
(\mathrm{MeV} / u)\end{array}$ & $\begin{array}{c}\text { Electron current } \\
(\mathrm{A})\end{array}$ & $\begin{array}{c}\epsilon_{H, V} \\
(\mathrm{~mm} \mathrm{mrad})\end{array}$ & $\Delta p / p$ \\
\hline 740 & 1 & $2 \times 10^{-2}$ & $5 \times 10^{-5}$ \\
100 & 1 & $6 \times 10^{-2}$ & $6 \times 10^{-5}$ \\
4 & 0.1 & 2 & $6 \times 10^{-4}$ \\
\hline \hline
\end{tabular}

\begin{tabular}{cccc}
\hline \hline \multicolumn{4}{c}{ Coasting beam of $10^{8}$ antiprotons } \\
\hline $\begin{array}{c}\text { Kinetic energy } \\
(\mathrm{MeV})\end{array}$ & $\begin{array}{c}\text { Electron current } \\
\text { (A) }\end{array}$ & $\begin{array}{c}\epsilon_{H, V} \\
(\mathrm{~mm} \text { mrad })\end{array}$ & $\Delta p / p$ \\
\hline 800 & 1.5 & $2 \times 10^{-3}$ & $2 \times 10^{-5}$ \\
30 & 0.5 & $5 \times 10^{-2}$ & $6 \times 10^{-5}$ \\
\hline \hline
\end{tabular}

corresponding space charge field induces an incoherent Laslett tune shift of $|\Delta Q| \simeq 0.1$, which is typically considered as the maximum acceptable value for the operation of the ring. This intensity limit at low energy is further reduced, if the beam is compressed into a short bunch before transfer to the FLAIR low energy experimental area.

\section{Rf SYSTEMS}

The operation of the NESR in various modes requires pertinent $\mathrm{rf}$ systems. At present, four types of rf systems are foreseen [1] (Fig. 2). Their main parameters are summarized in Table IV.

\section{A. Rf cavity for deceleration}

A ferrite-filled cavity with a frequency swing between 1 and $2.6 \mathrm{MHz}$ and a peak voltage of $15 \mathrm{kV}$ in cw operation will be installed, very similar to the existing SIS18 cavity. As the beams are cooled, the momentum spread is small and this voltage is sufficient for the fast ramp rate of $1 \mathrm{~T} / \mathrm{s}$. This cavity can decelerate all beams by changing the $\mathrm{rf}$ harmonic number in the course of the deceleration process between $h=2,4,8$. It will also allow debunching of single injected bunches in order to reach minimum momentum spread for fast cooling. If bunched beams are required for experiments this system can be used over the whole energy range by changing the harmonic number.

\section{B. Low frequency rf cavity}

A dedicated rf system operating at $h=1$ will compress the decelerated ion (at $4 \mathrm{MeV} / u$ ) or antiproton (at $30 \mathrm{MeV}$ ) beam into a bunch with a length of about $30 \mathrm{~m}$ as required (i) for transfer to a subsequent storage ring of the FLAIR facility or (ii) for injection into a trap after deceleration in a linac of the Highly charged Ion TRAPping facility (HITRAP) [8]. It consists of a singlegap resonator filled with magnetic alloy, with a frequency between 0.125 and $0.333 \mathrm{MHz}$ and a peak voltage of 1 and $5 \mathrm{kV}$ for $\mathrm{cw}$ and pulsed operation, respectively. After deceleration, the beam will be debunched and the resulting coasting beam will be adiabatically rebunched by this low frequency rf system. Then, in a second step, the bunch will be rotated in phase space by a quarter of a synchrotron oscillation period (within a mismatched bucket) and its length will be reduced to the required value for extraction.

TABLE IV. The rf systems of NESR.

\begin{tabular}{lccc}
\hline \hline Operation mode & Type & $\begin{array}{c}\text { Frequency } \\
(\mathrm{MHz})\end{array}$ & $\begin{array}{c}\text { Maximum voltage } \\
(\mathrm{kV})\end{array}$ \\
\hline Deceleration & ferrite filled & $1.0-2.6$ & 15 \\
Barrier bucket & broadband & 5, single sine & 2 \\
Low frequency & magnetic alloy & $0.125-0.333$ & $1(5$, pulsed $)$ \\
e-ion collider & $\lambda / 4$ cavity & $\approx 45$ & 20 \\
\hline \hline
\end{tabular}




\section{Barrier bucket system}

For beam accumulation at injection energy a broadband barrier bucket (BB) system is foreseen, which provides single sine waves of $200 \mathrm{~ns}$ period. Four cavities, each driven by a $3.5 \mathrm{~kW}$ solid state amplifier, result in a total voltage of $2 \mathrm{kV}$, which is sufficient to compress cooled beams (see Sec. VI).

According to recent studies, this system could also compress the decelerated beam into a bunch with a total $(4 \sigma)$ length of $30 \mathrm{~m}$ for transfer to FLAIR. Therefore, it might make the installation of the dedicated low frequency system dispensable. This is demonstrated in Fig. 7 for a beam of $10^{8}{ }^{238} \mathrm{U}^{92+}$ ions at $4 \mathrm{MeV} / u$. The ESME code [19] was used to simulate the rf system. An initial momentum (energy) spread of the cooled coasting beam of $6 \times 10^{-4}$ $\left(4.8 \times 10^{-3} \mathrm{MeV} / u\right)$ was assumed, in accordance with the results of simulations with BETACOOL (Table III). Two overlapping BB sine pulses of $2 \mu$ s period are adiabatically introduced into the beam. One stays stationary whereas the other is shifted in phase until the required bunch length is reached. For a maximum voltage of $2 \mathrm{kV}$, the corresponding bucket height of $3.9 \times 10^{-3}(3.1 \times$ $10^{-2} \mathrm{MeV} / u$ ) is sufficient to capture the beam. Similar simulations have been made for a $30 \mathrm{MeV}$ antiproton beam. For the antiprotons the situation in terms of rf voltage is more relaxed, because, due to their lower charge, lower momentum spreads are expected at the equilibrium between electron cooling and IBS. (The electron cooling rate scales with $Z^{2} / A$ whereas the IBS rate increases with $Z^{4} / A^{2}$.) Shorter compression times can be expected for special ramping conditions of the $\mathrm{BB}$ pulses and with continuous application of electron cooling during the compression.

In addition, both for ions and antiprotons, the BB system still offers the option of a bunch rotation at $h=1$ in order to produce a short bunch for extraction, as illustrated by the ESME simulations in Fig. 8. The cooled coasting beam with an assumed initial momentum (energy) spread of $6 \times 10^{-4}$ $\left(4.8 \times 10^{-3} \mathrm{MeV} / u\right)$, in accordance with the results of simulations with BETACOOL (Table III), is adiabatically captured within $100 \mathrm{~ms}$ by a sinusoidal rf wave at $h=1$. The rf bucket remains stationary for another $20 \mathrm{~ms}$. Then, the $\mathrm{rf}$ voltage is increased very fast $(\approx 100 \mathrm{~ns}$ ) to the peak value of $2 \mathrm{kV}$ and the bunch starts to rotate in the bucket. After $600 \mathrm{~ns}$, the bunch is rotated by $90^{\circ}$. For the users of the extracted beams and, in particular, for injection into the decelerating linac of the HITRAP facility, the compression is preferable because it leads to lower momentum spreads than the bunch rotation.

\section{High harmonics rf cavity for electron-ion collider mode}

For the operation of the NESR in the collider mode, a rf system operating at about $45 \mathrm{MHz}$ will provide short ion bunches matched to the relativistic electron bunches in the

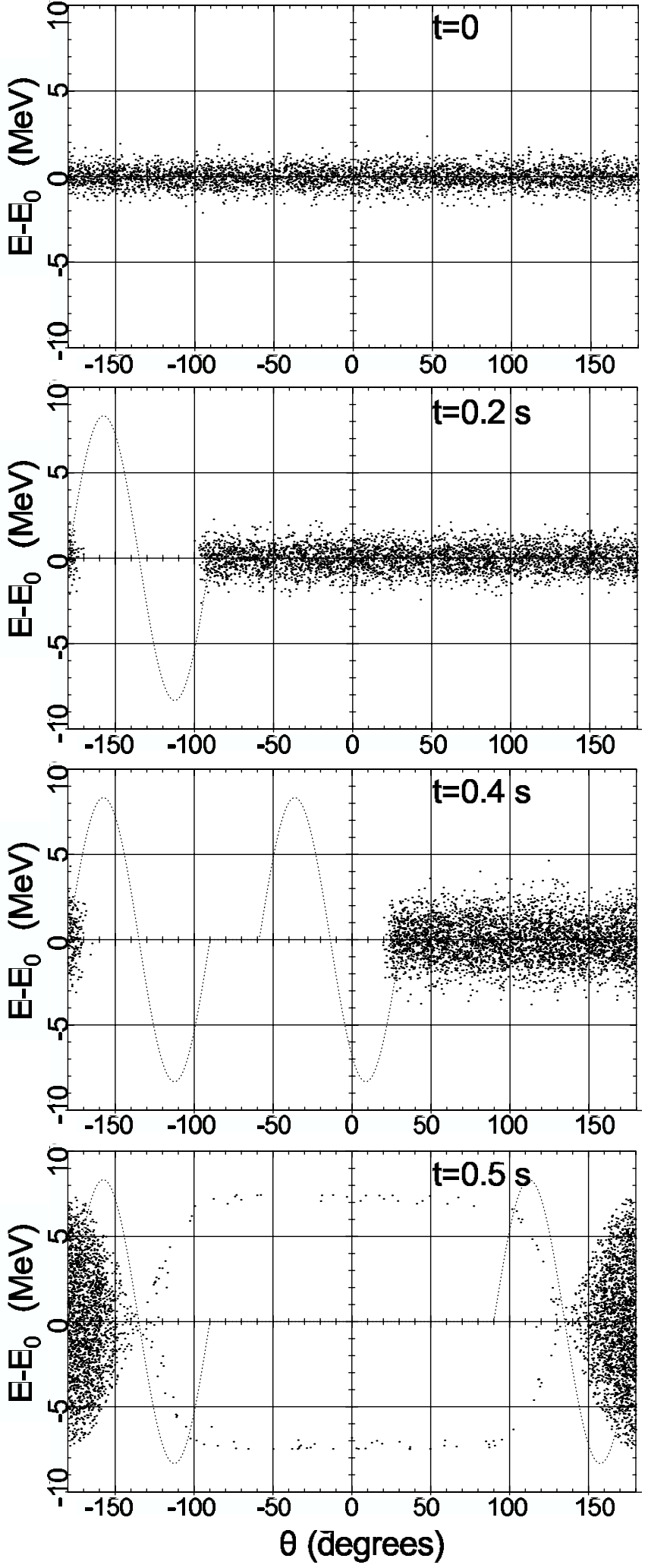

FIG. 7. Compression of the $4 \mathrm{MeV} / u{ }^{238} \mathrm{U}^{92+}$ beam into a bunch of $30 \mathrm{~m}$ by barrier bucket pulses which have a peak voltage of $2 \mathrm{kV}$ (dotted lines). The dots represent the particle distribution in the longitudinal phase space. No electron cooling is included in the ESME simulation. 

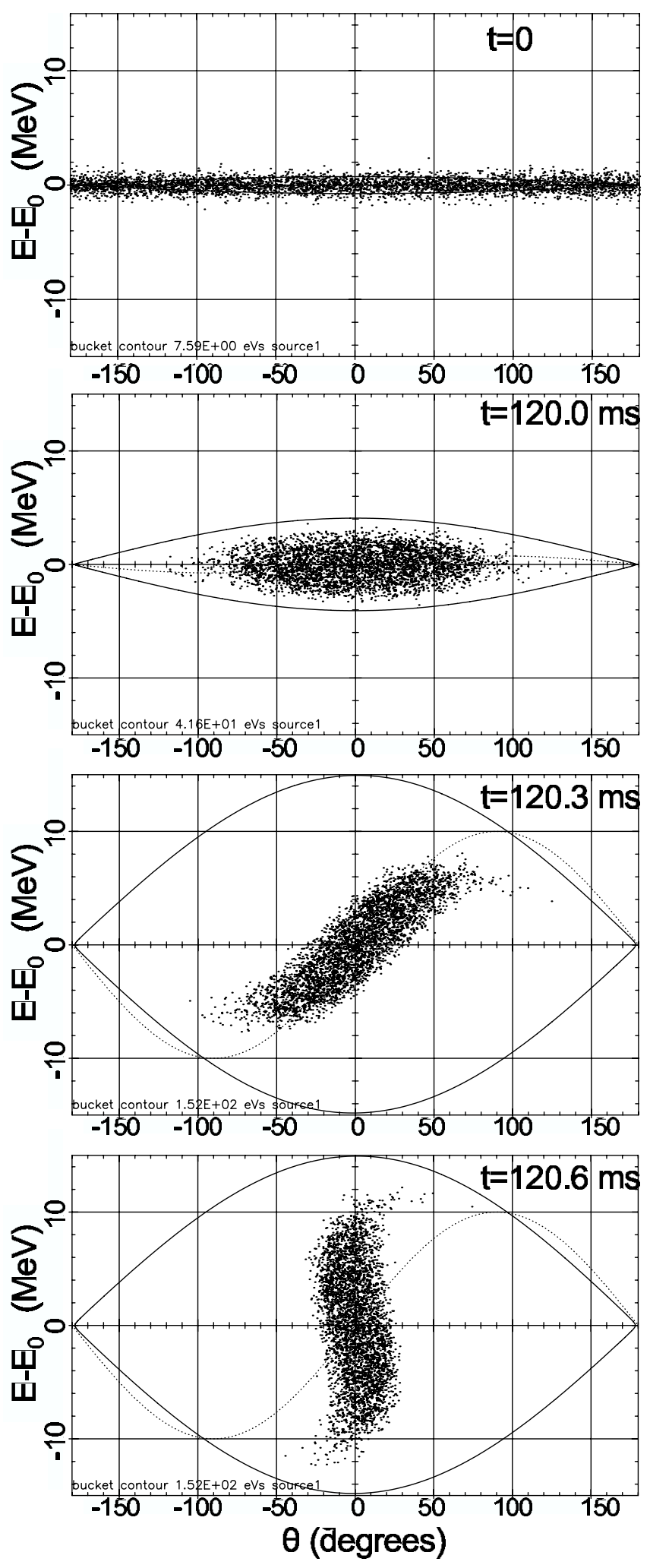

FIG. 8. Adiabatic capture and rotation of the $4 \mathrm{MeV} / u$ ${ }^{238} \mathrm{U}^{92+}$ bunch by applying sinusoidal rf signals (dotted lines) to the BB cavity. The dots represent the particle distribution in the longitudinal phase space. The rotation is made with a $2 \mathrm{kV}$ peak voltage and results in a bunch length $(4 \sigma)$ of $30 \mathrm{~m}\left(48^{\circ}\right)$. No electron cooling is included in the ESME simulation. electron storage ring. The parameters of this system will be defined depending on the design of the electron ring.

\section{ACCUMULATION OF RIBS AT INJECTION ENERGY}

In order to reach the high intensity of RIBs required by the internal experiments and, in particular, by the electronion collider mode, it is planned to stack the RIBs longitudinally at injection energy $(100-740 \mathrm{MeV} / u)$. The favored method of longitudinal beam accumulation is based on a barrier bucket $\mathrm{rf}$ system in combination with electron cooling. According to dedicated beam dynamics simulations [20], the stacking cycle time (i.e. the time between 2 successive injections) could be about $2 \mathrm{~s}$, provided that the quality of the injected precooled beam allows cooling times below $1 \mathrm{~s}$. With this stacking cycle time the experiments can take full advantage of the planned cycle time of $1.5 \mathrm{~s}$ of SIS100, where the primary heavy ion beam is accelerated. As an alternative, a $h=1 \mathrm{rf}$ system for bunching of the circulating beam and injection of a new bunch onto the unstable fixed point in longitudinal phase space is considered [21,22]. The rf voltage is raised so as to confine the bunch in a small fraction of the ring circumference. A new bunch is injected onto the free part of the circumference. Then the rf voltage is decreased to let the beam debunch. Continuous application of electron cooling maintains the stack and merges it with the freshly injected bunch. For both methods, the maximum useful accumulation time is limited by the lifetime of the ion beam, either due to nuclear decay or due to recombination with electrons of the cooling system.

\section{CONCLUSIONS}

The basic design and operation modes of the NESR ring have been presented. Clearly, the performance of the ring will depend not only on its optics and components, such as the electron cooling and rf systems, but also on the parameters of its injectors and, in particular, on the performance of the CR/RESR complex. In this respect, the storage rings of the FAIR facility are being designed as a whole. A number of issues is still under study. Additional input from the in-ring experiments as well as from the users of the extracted beams is required in order to optimize and finalize the details of the ring design.

\section{ACKNOWLEDGMENTS}

This article has benefited from the input of the late Peter Beller who has made essential contributions to this work. The authors are grateful to T. Katayama and D. Möhl for their studies on the stacking methods as well as for numerous fruitful discussions. We also thank B. Franzke for his useful comments. We acknowledge support from the EU 
design study (Contract No. 515873-DIRACsecondaryBeams).

[1] An International Accelerator Facility for Beams of Ions and Antiprotons, Conceptual Design Report, GSI, 2001 and Baseline Technical Design Report, GSI, 2006. Both reports are available at http://www.gsi.de/fair/reports/.

[2] P. Beller, K. Beckert, A. Dolinskii, B. Franzke, F. Nolden, and M. Steck, Proceedings of the 8th European Particle Accelerator Conference, Paris, 2002, p. 545.

[3] P. Beller et al., Proceedings of the 10th European Particle Accelerator Conference, Edinburgh, 2006, http:// accelconf.web.cern.ch/.

[4] J. Stadlmann et al., in Ref. [3].

[5] H. Geissel et al., Nucl. Instrum. Methods Phys. Res., Sect. B 204, 71 (2003).

[6] F. Nolden et al., in Ref. [3].

[7] E. Widmann, 6th International Conference on Nuclear Physics at Storage Rings, STORI05, Jülich-Bonn, Germany, 2005.

[8] SPARC Technical Report, GSI, 2006, http://www.gsi.de/ fair/experiments/sparc/.

[9] F. Sprenger et al., Nucl. Instrum. Methods Phys. Res., Sect. A 532, 298 (2004).
[10] I. A. Koop, P. V. Logatchev, I. N. Nesterenko, A. V. Otboev, V. V. Parkhomchuk, V.M. Pavlov, E. A. Perevedentsev, D. N. Shatilov, P. Yu. Shatunov, Yu. M. Shatunov, S. V. Shiyankov, A. N. Skrinsky, A. A. Valishev, P. Beller, B. Franzke, and M. Steck, Proceedings of the 8th European Particle Accelerator Conference, Paris, 2002, p. 620.

[11] P. Beller, K. Beckert, A. Dolinskii, B. Franzke, F. Nolden, C. Peschke, and M. Steck, Proceedings of the 9th European Particle Accelerator Conference, Lucerne, 2004, p. 1174.

[12] http://www-linux.gsi.de/redelbac/MIRKO/.

[13] http://frs.web.cern.ch/frs/.

[14] http://mad.web.cern.ch/mad/.

[15] V. V. Parkhomchuk et al., GSI-Acc-Report-2005-04-001.

[16] A. O. Sidorin, I. N. Meshkov, I. A. Seleznev, E. M. Syresin, and G. V. Trubnikov, Nucl. Instrum. Methods Phys. Res., Sect. A 558, 325 (2006).

[17] V. V. Parkhomchuk, Nucl. Instrum. Methods Phys. Res., Sect. A 441, 9 (2000).

[18] M. Martini, CERN-PS-84-9, 1984.

[19] http://www-ap.fnal.gov/ESME/.

[20] T. Katayama (private communication); (unpublished).

[21] D. Möhl (private communication); (unpublished).

[22] S. Baird et al., IEEE Proceedings of the Particle Accelerator Conference, 1989, p. 645. 